\title{
PARTICULAR BIOLOGICAL MODEL OF THERAPEUTIC RESISTANCE IN THE ONCOLOGICAL PATIENT WITH DEPRESSION
}

\section{Marinescu ${ }^{1}$ D. Marinescu ${ }^{1}$, R.A. Schenker ${ }^{2}$, D.G. Malaescu ${ }^{3}$, P.O. Stovicek \\ ${ }^{1}$ University of Medicine and Pharmacy of Craiova, Department of Psychiatry, Craiova, Romania \\ ${ }^{2}$ University of Medicine and Pharmacy of Craiova, Doctoral School, Craiova, Romania \\ 3“Titu Maiorescu” University- Bucharest, Department of Morphopathology, Craiova, Romania \\ 4"Titu Maiorescu” University- Bucharest, Department of Pharmacology, Craiova, Romania}

\section{NEUROTOXIC MECHANISMS}

MECHANISMS INDUCED BY ONCOLOGICAL TREATMENT $(1,2)$

Anthracyclines

Bleomycin

Mitomycin C

Platinum salts

Vincristine

Paclitaxel

Radiotherapy
NEUROSTRUCTURAL VULNERABILITIES INDUCED BY DEPRESSION

- Oxidative stress
- Cytokine mechanisms
- Immune mechanisms
- Excitotoxicity
- Neural apoptosis

\section{DEPRESSION ENHANCES NEUROTOXIC MECHANISMS INDUCED BY ONCOLOGICAL MEDICATION}

Figure 1. Neurotoxic mechanisms in oncological patients and depression

\section{Introduction:}

Due to high depression rate in oncology (40-48\%), psychiatric therapeutic approach is an important target in oncology management. Objectives:

Oncological chemotherapy works frequently through neurotoxic mechanisms (platinum based agents) (figure 1). Also, radiotherapy treatment causes atrophy of hipocampus, thalamus and prefrontal cortex. Combining chemotherapy with radiotherapy increases cognitive disconnectivity by altering hippocampal-frontal circuits.

\section{Methods:}

Alteration of hippocampal structure was demonstrated on animal model (rat) by highlighting neuronal necrosis and vacuolization of white matter (figure 2). From clinical perspective, hippocampal neurotoxic syndrome associates mild cognitive impairment.

\section{Results:}

Disconnectivity to the thalamic structure increases pain sensitivity and a loss of functional integrity with cerebral amygdala, resulting in impulsivity-aggressiveness behavioral disorders, up to suicidal behavior.

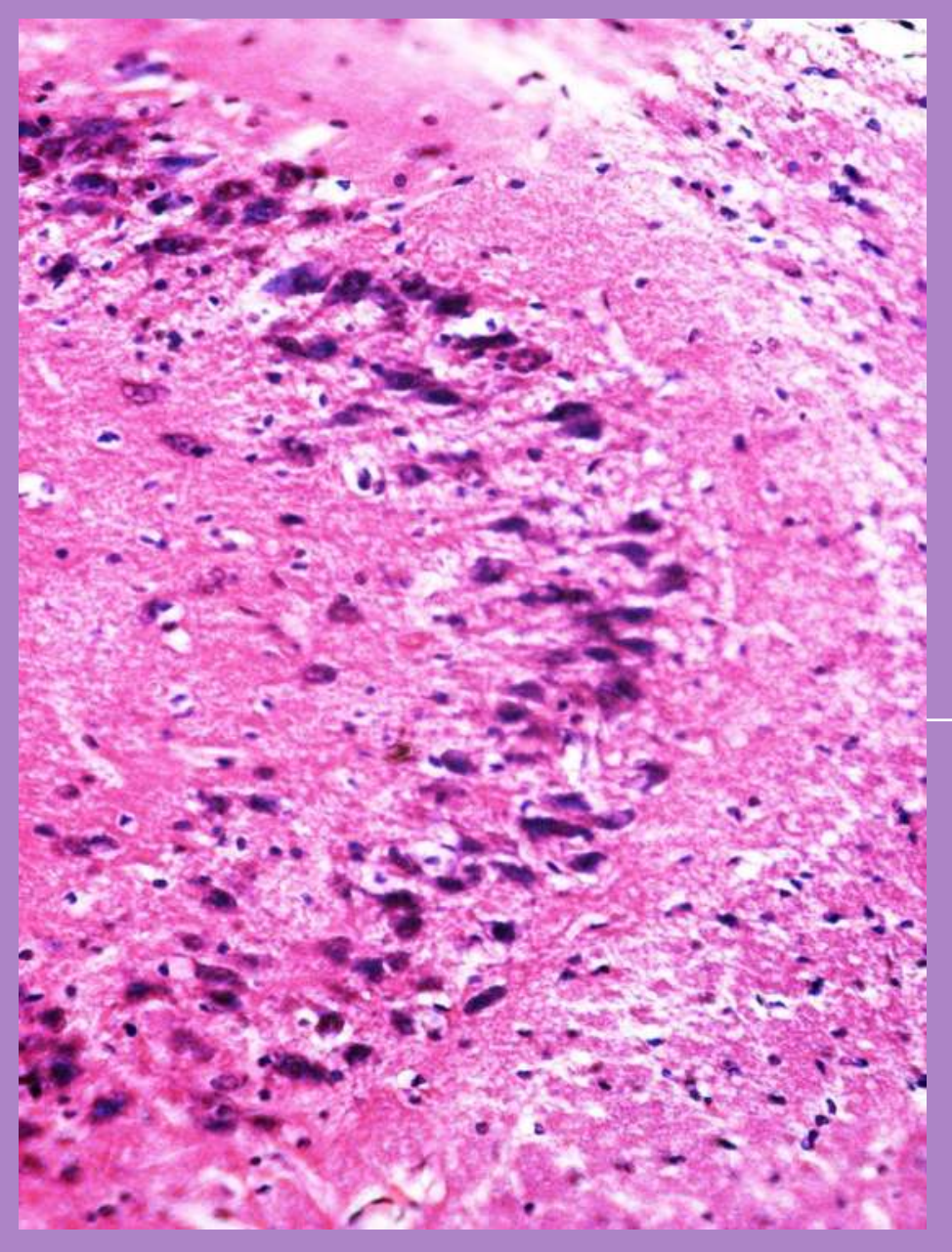

Figure 2. Experimental model on Wistar rat after administration of dexametahasone, 7 days, Hematoxylineosin staining, Objective x20. Cellular alterations of the

hippocampal structure with neuronal necrosis and apoptosis, white matter vacuolar degeneration
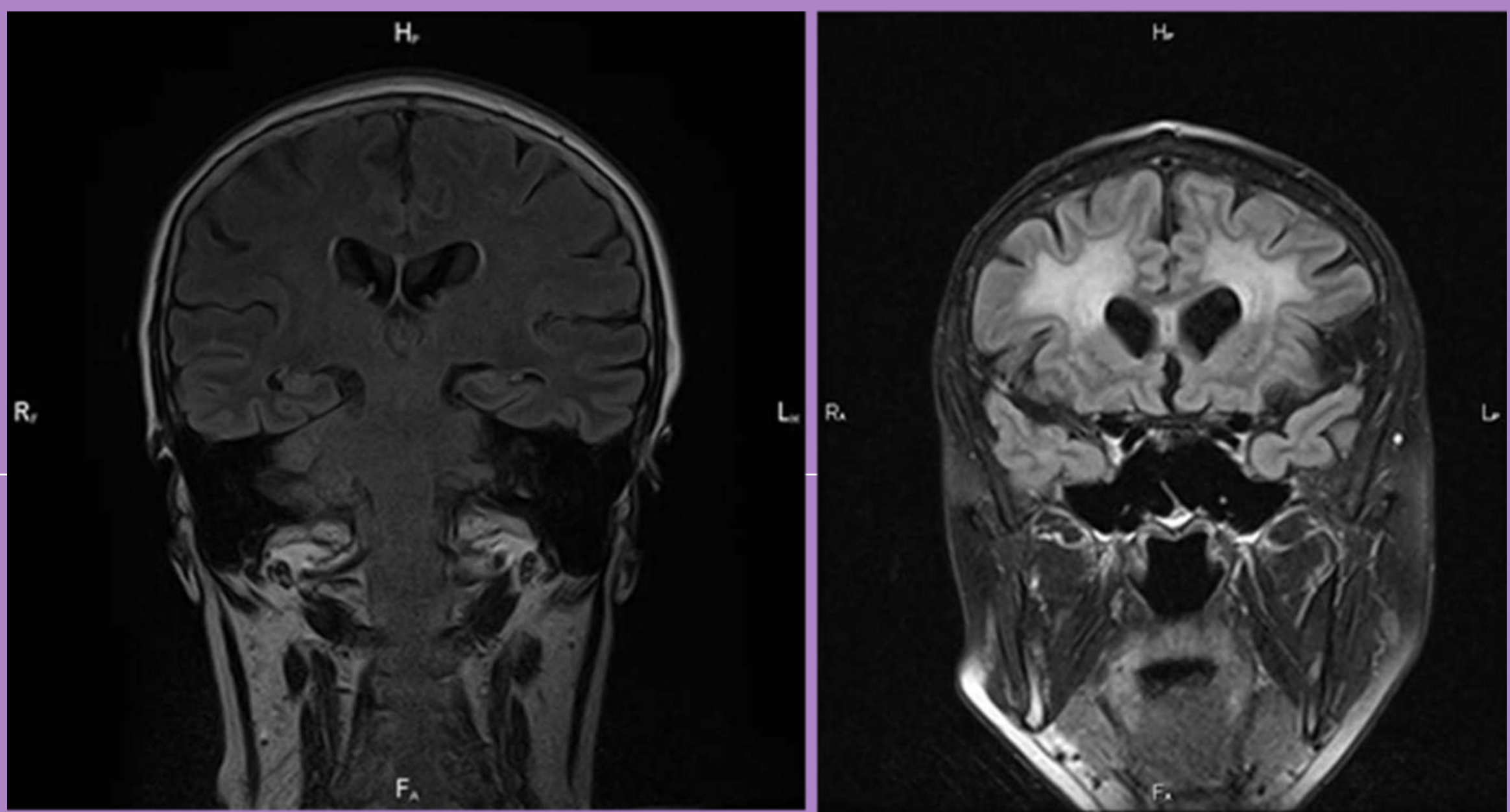

Figure 3. The 60-year-old patient with depression and cervical cancer treated with radiotherapy and chemotherapy

1. (Left) Pretreatment MRI: Cerebral substance within the age range, with no pericallosal demyelination, no cerebral atrophy, symmetrical hippocampal gyres without modification. 2. (Right) MRI exam after 2 years of treatment: marked degenerative changes, translated into areas of hypersensitive Flair with pericallosal appearance of degenerative aspect, reactive gliosis and dilatation of Virchow-Robin spaces, right side hippocampus atrophy

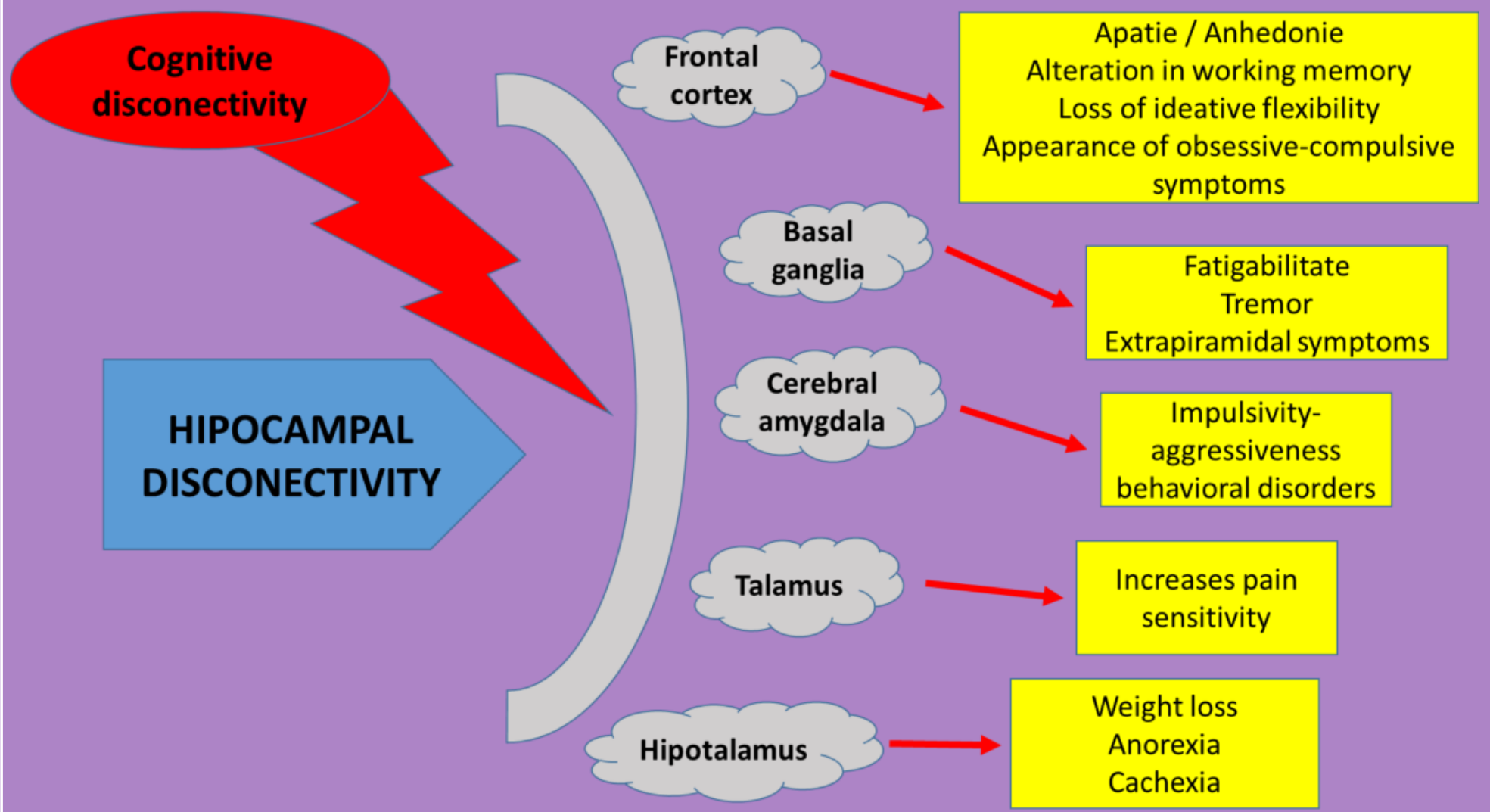

Figure 4. The effects of hippocampal disconnectivity and cognitive impairment

References:

1. Noriko Noda, Hiro Wakasugi, Cancer and Oxidative Stress, JMAJ 44(12): 535-539, 2001 2. Amteshwar Singh Jaggi, Nirmal Singh, Mechanisms in cancer-chemotherapeutic drugsinduced peripheral neuropathy, Toxicology 291 (2012) 1-9.
Hippocampal and thalamic atrophy during chemotherapy or radiotherapy (figure 3) represent an indirect suicide risk marker of in oncological disease. Atrophy of the frontal cortex causes changes in working memory, loss of ideative flexibility and the appearance of obsessive-compulsive disorder symptoms (figure 4). These mechanisms following neurotoxic effect of oncology-specific treatment can be amplified by dopamine-blocking psychotropic medication (D2 blocking antipsychotics or serotoninergic activators). This pharmacological mechanism increases the oxidative stress in the described cognitive structures and amplifies neuronal excitotoxicity through a high glutamate level, ultimately causing apoptosis. Conclusions:

The described mechanisms can be an evolutionary risk model in the oncology patient with depression, in which the specific oncology therapy, the psychotropic medication and the cognitive-behavioral psychotherapeutic interventions, can not benefit from an adequate therapeutic response (secondary therapeutic resistance). Neuroprotection and stimulation of cognitive circuits are required by alternative means (physical exercise, melotherapy, non-invasive methods for interhemispheric reconnection). 